RESEARCH NOTE

\section{Random by Amplified Polymorphic DNA Analysis of Sylvatic Trypanosoma cruzi Isolates Infers from French Guiana Accurate Phylogeny}

\author{
Brigitte Bastrenta $^{+}$, Simone \\ Frédérique Brenière
}

ORSTOM UR 7 "Transmission, Expression, Prévention et Contrôle des Maladies à Vecteurs", BP 5045, 34032 Montpellier cedex 1, France

Key words: Trypanosoma cruzi - sylvatic cycle RAPD - synapomorphic character

Trypanosoma cruzi, the agent of Chagas disease, exhibits considerable genetic diversity as evidenced by multilocus enzyme electrophoresis (MLEE) and presents a basically clonal structure (MA Miles et al. 1978 Nature 272: 819-821, AJ Romanha et al. 1979 Comp Biochem Physio 62B: 139-142, M Tibayrenc et al. 1986 Proc Natl Acad Sci USA 83: 115-119). Clonality in T. cruzi has been mainly explored in domestic cycles. Nevertheless, in sylvatic cycles the possibility of genetic exchange could be more frequent (HJ Carrasco et al. 1996 Am J Trop Med Hyg 54: 418-424). However, recent MLEE analysis of wild T. cruzi stocks from French Guiana suggested that these sylvatic populations are basically clonal too (K Lewicka et al. 1995 Exp Parasitol 81: 20-28). Moreover, a previous study of several species of parasites showed that random amplified polymorphic DNA (RAPD) was a suitable tool for evolutionary studies in pathogenic microorganisms (M Tibayrenc et al. 1993 Proc Nat Acad Sci USA 90: 1335-1339, M Steindel et al. Mol Biochem Parasitol 60: 71-80).

In order to explore further population structure in wild $T$. cruzi stocks, 26 trypanosomatid stocks isolated in French Guiana, previously characterized

This work was supported by a WHO TDR grant no. 880190 .

${ }^{+}$Corresponding author. Present address: ORSTOM Mission Bolivie, CP 9214, La Paz, Bolivia. Fax: 591239 14 16. E-mail: Bastrenta@ns.megalink.com

Received 3 November 1997

Accepted 12 March 1998 by MLEE, were studied by means of RAPD (11 10mer primers: A1, A4, A7, A8, A9, A10, A12, A17, A18, A19, A20, Operon Technologies) (Tibayrenc loc. cit., JGK Williams 1990 Nucleic Ac Res 18: 6531-6535). The host origin from which the isolates were obtained, is given in Lewicka et al. (loc. cit.). Four reference stocks: Tehuantepec cl1, CanIII cl1 Z3, SC43 cl2, Tulahuen FKIIA, were included to overall T. cruzi genetic variability (M Tibayrenc \& FJ Ayala 1988 Evolution 42: 277-292).

Each RAPD assay presented a limited number of bands and only the reproducible bands were retained. Each primer generates polymorphic patterns except the A20 primer. Among a total of 93 scored bands, 74 were observed within the Guianese sample. Twenty two different multilocus RAPDemes were obtained and the genotype diversity was therefore $22 / 26=0.84$. This rate of genotype diversity was similar to the one obtained from MLEE data (0.78) (Lewicka et al. loc. cit.). Most of these RAPDemes were differentiated by the A1 single primer (16 different genotypes), though the nine other polymorphic primers showed an average of 5.1 genotypes.

Phylogenetic relationships among the stocks were analyzed by computed dendrogram from the Jaccard's distance matrix using the UPGMA (unweighted pair-group method with arithmetic averages) algorithm (Fig. 1) (P Jaccard 1908 Bull Soc Vaudoise Sci Nat 44: 223-270). Most Guianese stocks belong to the same cluster (Fig. arrow) that occurred 90.4 times out of 100 replicates (Bootstrap analysis, PHYLIP program, J Felseinstein 1978 Systematic Zoology 27: 27-33). This group includes the Tehuantepec reference stock pertaining to the formally described Zymodeme I (Miles loc. cit.). Previous analysis on the same set of stocks, by MLEE (Lewicka et al. loc. cit.), gave a similar dendrogram topology and both markers (RAPD and MLEE) are statistically correlated (Mantel test, $\left.\mathrm{p}<10^{-4}\right)(\mathrm{N}$ Mantel 1967 Canc Res 27: 209-220). This result strongly suggests that this cluster corresponds to a monophyletic group. MLEE analysis clustered three stocks, namely, A83, A87 and A276, apart from T. cruzi reference stocks and the Guianese sample. The current RAPD analysis showed that A83 stock is related to Tehuentepec reference stock (Jaccard's distance of 0.5) (Fig. 1), while, A87 and A276 stocks present a high genetic distance to the other stocks. A recent study suggested that A8 primer generates a specific single band in T. cruzi species that is absent from closely related species (B Oury $1996 J$ Parasitol 83: 52-57). This band was shared by the four T. cruzi reference stocks, and all Guianese stocks except A276. Moreover, one of the bands, generated by A4 primer, showed an identical distribution among the stocks and should be considered 


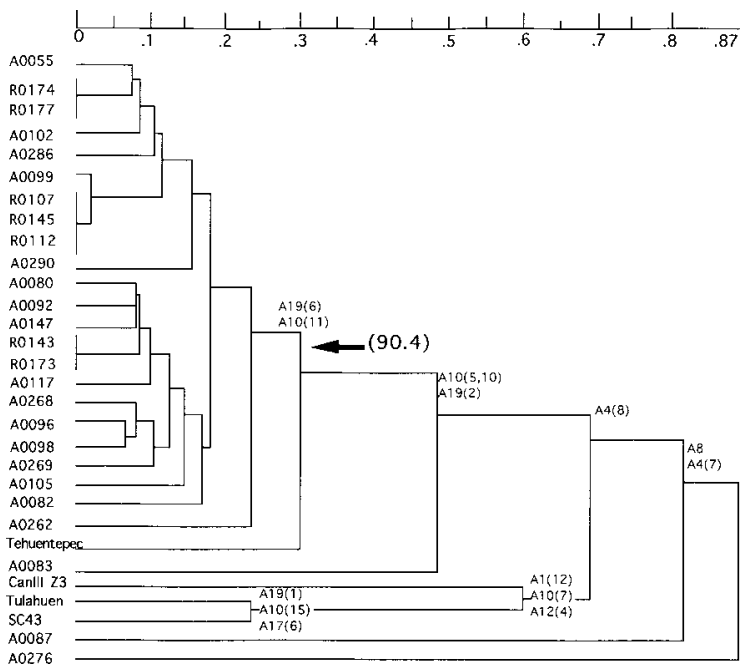

Fig. 1: dendrogram of the 26 trypanosomatid stocks isolated in French Guiana and 4 Trypanosoma cruzi reference stocks, constructed by UPGMA from a Jaccard's distance matrix, obtained by RAPD. The arrow indicates the principal cluster with the Bootstrap value. The synapomorphic characters are labeled with a number that indicates the primer used and the number in parentheses refers to a particular band.

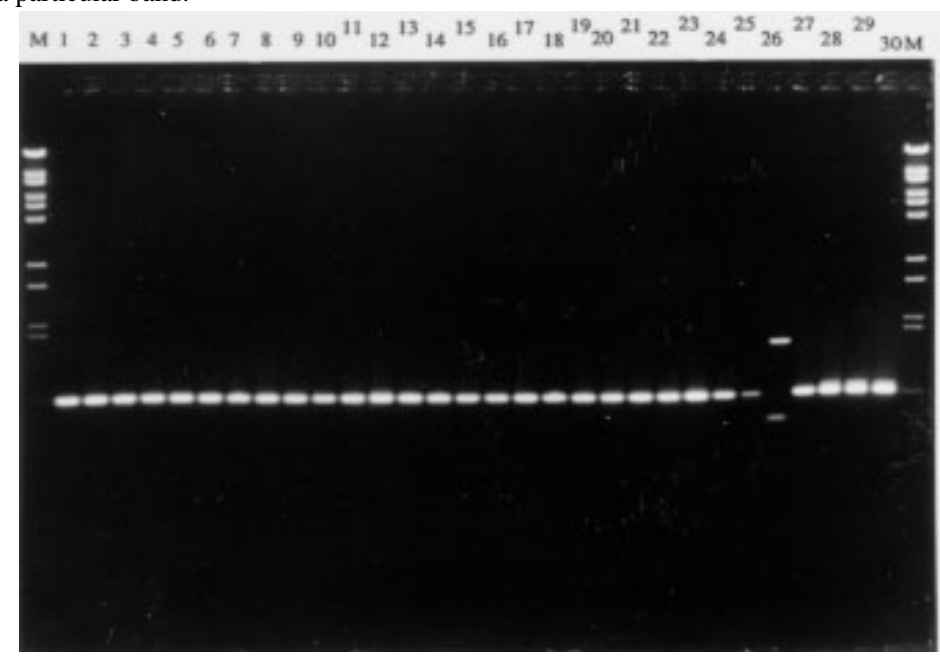

Fig. 2: one-banded monophorphic RAPD patterns generated by the A8 primer. Lane 1 to 26: Trypanosoma cruzi Guianese stocks: A0055, A0080, A0092, A0099, R0107, R0145, R0112, R0143, A0105, A0117, A0147, A0102, R0174, R0177, R0173, A0096, A0098, A0286, A0290, A0268, A0269, A0262, A0082, A0083, A0087, A0276. Lane 27 to 30: T. cruzi reference stocks: Tehuantepec cl1, Tulahuen FKIIA, CanIII cl1 Z3, SC43 cl2. M: molecular weigthmarker (Bst E II-digested l phage).

also as a specific character of $T$. cruzi taxon. Considering these two taxonomic markers, the $\mathrm{A} 83$ and A87 stocks pertain to $T$. cruzi taxon, although the last one presents a high genetic distance from the other stocks. The taxonomic position of A276 stock is more likely apart from $T$. cruzi taxon.

Fig. 2 indicates at internal nodes of the tree, the bands for each primer shared by the group above and to the right of that node. These bands should be equated to synapomorphic characters and accurate to the current observed divisions. The absence of synapomorphic character at the lower level of divergence makes more questionable the relationship between closely related stocks. A broader range of RAPD primers should be convenient to study genetically related stocks.

In conclusion, the advantage of RAPD analysis above MLEE is to generate potential markers that could be used as diagnostic tools for delimitation of $T$. cruzi species and specific of phylogenetic subdivisions.

Acknowledgments: to Professor Jean-Pierre Dedet (Faculté de Médecine, Montpellier, France) for providing the T. cruzi isolates from French Guiana, Christian Barnabé (ORSTOM, Montpellier, France) for processing the bootstrap analysis and Michel Tibayrenc (ORSTOM, Montpellier, France) for helpful comments on the manuscript. 